\title{
Multiplicity results on discrete boundary value problems with double resonance via variational methods
}

\section{Xiaosheng Zhang*}

\section{"Correspondence:}

zhangxs@cnu.edu.cn

School of Mathematical Sciences,

Capital Normal University, Beijing,

100048, China

\begin{abstract}
The existence of solutions for a class of difference equations with double resonance is studied via variational methods, and multiplicity results are derived.
\end{abstract}

Keywords: discrete boundary value problem; double resonance; existence and multiplicity; variational method

\section{Introduction}

Let $\mathbf{Z}$ denote the set of integers, and for $a, b \in \mathbf{Z}$ with $a<b$, define $\mathbf{Z}[a, b]=\{a, a+1, \ldots, b\}$. For a given positive integer $N \geq 2$, consider the following discrete boundary value problem:

$$
\left\{\begin{array}{l}
-\Delta^{2} x(k-1)=f(k, x(k)), \quad k \in \mathbf{Z}[1, N], \\
x(0)=x(N+1)=0,
\end{array}\right.
$$

where $\Delta$ is the forward difference operator defined by $\Delta x(k)=x(k+1)-x(k)$ and $\Delta^{2} x(k)=$ $\Delta(\Delta x(k))$ for $k \in \mathbf{Z}$. Throughout this paper, we always assume that $f: \mathbf{Z}[1, N] \times \mathbf{R} \rightarrow \mathbf{R}$ is $C^{1}$-differentiable with respect to the second variable and satisfies $f(k, 0) \equiv 0$ for $k \in$ $\mathbf{Z}[1, N]$, which implies that (BP) has a trivial solution $x(k)=0, k=0,1, \ldots, N+1$. We investigate the existence of nontrivial solutions of (BP).

In different fields of research, such as computer science, mechanical engineering, control systems, population biology, economics and many others, the mathematical modeling of important questions leads naturally to the consideration of nonlinear difference equations. The dynamic behaviors of nonlinear difference equations have been studied extensively in $[1,2]$. Recently, many authors considered the solvability of nonlinear difference equations via variational methods. For example, on the second-order difference equations, the boundary value problems are studied in [3-7] and the existence of periodic solutions is investigated in [8-10].

As a natural phenomenon, resonance exists in the real world from macrocosm to microcosm. In a system described by a mathematical model, the feature of resonance lies in the interaction between the linear spectrum and the nonlinearity. It is known from [1] that 
the eigenvalue problem

$$
\left\{\begin{array}{l}
-\Delta^{2} x(k-1)=\lambda x(k), \quad k \in \mathbf{Z}[1, N] \\
x(0)=x(N+1)=0
\end{array}\right.
$$

possesses $N$ distinct eigenvalues $\lambda_{l}=4 \sin ^{2}(l \pi / 2(N+1)), l=1,2, \ldots, N$. Many authors considered the complete resonance situation in the sense that for some $h \in \mathbf{Z}[1, N]$,

$$
\lim _{|t| \rightarrow \infty} \frac{f(k, t)}{t}=\lambda_{h}, \quad k \in \mathbf{Z}[1, N]
$$

via different methods in critical point theory such as Morse theory [6], index theory [9] and minimax methods [7]. The assumption that

$\left(f_{\infty}^{*}\right)$ there exists some $h \in \mathbf{Z}[1, N-1]$ such that

$$
\lambda_{h} \leq \liminf _{|t| \rightarrow \infty} \frac{f(k, t)}{t} \leq \limsup _{|t| \rightarrow \infty} \frac{f(k, t)}{t} \leq \lambda_{h+1} \quad \text { for } k \in \mathbf{Z}[1, N]
$$

characterizes problem (BP) as double resonance between two consecutive eigenvalues at infinity. In the case of resonance, one needs to impose various conditions on the nonlinearity of $f$ near infinity to ensure the global compactness. In fact, many results on differential equations with double resonance have been obtained (see [11-13]). As to discrete boundary value problems with double resonance, however, there are few results published. In [10], the existence of periodic solutions to a second-order difference equation with double resonance, as is described in $\left(\mathrm{f}_{\infty}^{*}\right)$, is investigated.

Motivated by the study in [10], we consider problem (BP) with double resonance indicated in $\left(f_{\infty}^{*}\right)$. To control the double resonance, a selectable restriction on the nonlinearity of $f$ is that

$\left(\mathrm{f}_{\infty}^{\dagger}\right)$ there exists some $h \in \mathbf{Z}[1, N-1]$ such that

$$
\begin{aligned}
& \text { (i) } \underline{r}_{h}(f):=\liminf _{|t| \rightarrow \infty}|t|\left(\frac{f(k, t)}{t}-\lambda_{h}\right)>0 \\
& \text { (ii) } \bar{r}_{h}(f):=\limsup _{|t| \rightarrow \infty}|t|\left(\frac{f(k, t)}{t}-\lambda_{h+1}\right)<0
\end{aligned}
$$

which has completely the same form as its counterpart in [10]. However, instead of $\left(f_{\infty}^{\dagger}\right)$, in this paper we assume that

$\left(\mathrm{f}_{\infty}\right)$ there exists some $h \in \mathbf{Z}[1, N-1]$ such that

$$
\begin{aligned}
& \text { (i) } \underline{R}_{h}(f):=\liminf _{|t| \rightarrow \infty} t^{2}\left(\frac{f(k, t)}{t}-\lambda_{h}\right)>0 \\
& \text { (ii) } \bar{R}_{h}(f):=\limsup _{|t| \rightarrow \infty} t^{2}\left(\frac{f(k, t)}{t}-\lambda_{h+1}\right)<0
\end{aligned}
$$

Remark 1.1 It is easy to see that, as a restriction on the nonlinearity of $f,\left(\mathrm{f}_{\infty}\right)$ is more relaxed than $\left(f_{\infty}^{\dagger}\right)$ (see Examples 1.1-1.3 and Remark 1.3). In addition, $\left(f_{\infty}\right)$, as well as $\left(f_{\infty}^{\dagger}\right)$, implies $\left(f_{\infty}^{*}\right)$. 
A sequence $\{x(0), x(1), \ldots, x(N+1)\}$ is said to be a positive (negative) solution of (BP) if it satisfies (BP) and $x(k)>0(<0)$ for $k \in \mathbf{Z}[1, N]$.

Theorem 1.1 Assume that $\left(\mathrm{f}_{\infty}\right)$ holds. Then (BP) has at least four nontrivial solutions in which one is positive and one is negative in each of the following two cases:

(i) $h \in \mathbf{Z}[2, N-1]$ and $f^{\prime}(k, 0)<\lambda_{1}$ for $k \in \mathbf{Z}[1, N]$;

(ii) $h \in \mathbf{Z}[1, N-2]$ and $f^{\prime}(k, 0)>\lambda_{N}$ for $k \in \mathbf{Z}[1, N]$.

To state the following theorems, we further assume that

$\left(\mathrm{f}_{0}\right)$ there exists $t_{0} \neq 0$ such that $f\left(k, t_{0}\right)=0$ for $k \in \mathbf{Z}[1, N]$.

Theorem 1.2 Assume that $\left(\mathrm{f}_{0}\right)$ and $\left(\mathrm{f}_{\infty}\right)$ hold with $h \in \mathbf{Z}[2, N-1]$. If there exists $m \in$ $\mathbf{Z}[2, N-1]$ with $m \neq h$ such that $\lambda_{m}<f^{\prime}(k, 0)<\lambda_{m+1}$ for $k \in \mathbf{Z}[1, N]$, then (BP) has at least four nontrivial solutions.

Let $f^{\prime}(k, t)$ denote the derivative of $f(k, t)$ with respect to the second variable. In the case where (BP) is also resonant at the origin, that is, there exists $m \in \mathbf{Z}[1, N]$ such that $f^{\prime}(k, 0) \equiv \lambda_{m}$ for $k \in \mathbf{Z}[1, N]$, we assume that

$\left(\mathrm{F}_{0}^{ \pm}\right) \quad \pm \int_{0}^{t}\left(f(k, s)-\lambda_{m} s\right) d s \geq 0 \quad$ for $|t|>0$ small and $k \in \mathbf{Z}[1, N]$.

Theorem 1.3 Assume that $\left(\mathrm{f}_{0}\right)$ and $\left(\mathrm{f}_{\infty}\right)$ hold with $h \in \mathbf{Z}[2, N-1]$. If there exists $m \in$ $\mathbf{Z}[1, N]$ such that $f^{\prime}(k, 0) \equiv \lambda_{m}$ for $k \in \mathbf{Z}[1, N]$, then (BP) has at least four nontrivial solutions in each of the following two cases:

(i) $\left(\mathrm{F}_{0}^{+}\right)$with $m \geq 2$ and $m \neq h$;

(ii) (F $\mathrm{F}_{0}^{-}$with $m \geq 3$ and $m \neq h+1$.

Remark 1.2 In view of the proofs in Section 4 , we see that if $t_{0}>0(<0)$ in $\left(f_{0}\right)$, two of the solutions derived in Theorems 1.2, 1.3 are positive (negative).

Set $h \in \mathbf{Z}[1, N-1]$ and define $g: \mathbf{R} \mapsto \mathbf{R}$ by

$$
g(t)=\lambda_{h} t+\left(\lambda_{h+1}-\lambda_{h}\right) t\left(1+t^{2}\right)^{-1}\left(\sin ^{2} t+t^{2} \cos ^{2} t\right) .
$$

By calculation, we get $\underline{R}_{h}(g)=-\bar{R}_{h}(g)=\lambda_{h+1}-\lambda_{h}>0$ and $g^{\prime}(0)=\lambda_{h}$. Define $g_{1}(t)=g(t)+$ $\alpha t\left(1+t^{4}\right)^{-1}, g_{2}(t)=g(t)+\left(\alpha t+\beta t^{2}\right)\left(1+t^{4}\right)^{-1}$ and $g_{3}(t)=g(t)+\left(\alpha t+\beta t^{3}\right)\left(1+t^{6}\right)^{-1}, t \in \mathbf{R}$, where $\alpha$ and $\beta$ are constants. Obviously, $\underline{R}_{h}\left(g_{i}\right)=\underline{R}_{h}(g)>0, \bar{R}_{h}\left(g_{i}\right)=\bar{R}_{h}(g)<0$ and $g_{i}^{\prime}(0)=\lambda_{h}+\alpha$, $i=1,2,3$. The following examples are presented to illustrate the applications of the above results.

Example 1.1 Consider (BP) with $f(k, t) \equiv g_{1}(t),(k, t) \in \mathbf{Z}[1, N] \times \mathbf{R}$. We have $f^{\prime}(k, 0)=$ $\lambda_{h}+\alpha$ for $k \in \mathbf{Z}[1, N]$. If $h \in \mathbf{Z}[2, N-1]$ and $\alpha<\lambda_{1}-\lambda_{h}$ or $h \in \mathbf{Z}[1, N-2]$ and $\alpha>\lambda_{N}-\lambda_{h}$, then by Theorem 1.1, (BP) has at least four nontrivial solutions in which one is positive and one is negative.

Example 1.2 Set $h, m \in \mathbf{Z}[2, N-1]$ with $m \neq h$. Let $\alpha \in\left(\lambda_{m}-\lambda_{h}, \lambda_{m+1}-\lambda_{h}\right)$ and $\beta<-\left(\lambda_{h}+\right.$ $\left.\lambda_{h+1}+\alpha\right)$. Consider (BP) with $f(k, t) \equiv g_{2}(t),(k, t) \in \mathbf{Z}[1, N] \times \mathbf{R}$. We have $f^{\prime}(k, 0)=\lambda_{h}+\alpha>$ 
$\lambda_{m}>0$ and $f(k, 1)=\left(\lambda_{h}+\lambda_{h+1}+\alpha+\beta\right) / 2<0$ for $k \in \mathbf{Z}[1, N]$, which implies that there exists $t_{0} \in(0,1)$ such that $f\left(k, t_{0}\right)=0$ for $k \in \mathbf{Z}[1, N]$. By Theorem 1.2 and Remark 1.2, (BP) has at least four nontrivial solutions in which two are positive.

Example 1.3 Set $h \in \mathbf{Z}[2, N-1], \alpha=\lambda_{m}-\lambda_{h}$ and $\beta<-\max \left\{\left(\lambda_{m}+\lambda_{h+1}\right), 2\left(\lambda_{h+1}-\lambda_{h}\right)\right\}$ for some $m \in \mathbf{Z}[3, N] \backslash\{h+1\}$. Consider (BP) with $f(k, t) \equiv g_{3}(t),(k, t) \in \mathbf{Z}[1, N] \times \mathbf{R}$. We have $f^{\prime}(k, 0)=\lambda_{h}+\alpha=\lambda_{m}>0$ and $f(k, 1)=\left(\lambda_{m}+\lambda_{h+1}+\beta\right) / 2<0$ for $k \in \mathbf{Z}[1, N]$, which implies that there exists $t_{0} \in(0,1)$ such that $f\left(k, t_{0}\right)=0$ for $k \in \mathbf{Z}[1, N]$. Moreover,

$$
\int_{0}^{t}\left(f(k, s)-\lambda_{m} s\right) d s=\left(\frac{\beta}{4}+\frac{\lambda_{h+1}}{2}-\frac{\lambda_{h}}{2}\right) t^{4}+o\left(t^{4}\right) \quad(t \rightarrow 0) .
$$

By Theorem 1.3(ii) and Remark 1.2, (BP) has at least four nontrivial solutions in which two are positive.

Remark 1.3 It is easy to see that $\underline{r}_{h}\left(g_{i}\right)=\bar{r}_{h}\left(g_{i}\right)=0, i=1,2,3$, that is, the restriction imposed here is more relaxed than that in [10].

The paper is organized as follows. In Section 2 we give a simple revisit to Morse theory, and in Section 3 we give some lemmas. The main results will be proved in Section 4.

\section{Preliminary results on critical groups}

Let $H$ be a Hilbert space and $\Phi \in C^{2}(H, \mathbf{R})$ be a functional satisfying the Palais-Smale condition ((PS) in short), that is, every sequence $\left\{x_{n}\right\} \subset H$ such that $\left\{\Phi\left(x_{n}\right)\right\}$ is bounded and $\Phi^{\prime}\left(x_{n}\right) \rightarrow 0$ as $n \rightarrow \infty$ has a convergent subsequence. Denote by $H_{q}(X, Y)$ the $q$ th singular relative homology group of the topological pair $(X, Y)$ with integer coefficients. Let $u_{0}$ be an isolated critical point of $\Phi$ with $\Phi\left(u_{0}\right)=c, c \in \mathbf{R}$, and $U$ be a neighborhood of $u_{0}$. For $q \in \mathbf{N} \cup\{0\}$, the group

$$
C_{q}\left(\Phi, u_{0}\right):=H_{q}\left(\Phi^{c} \cap U, \Phi^{c} \cap U \backslash\left\{u_{0}\right\}\right)
$$

is called the $q$ th critical group of $\Phi$ at $u_{0}$, where $\Phi^{c}=\{u \in H: \Phi(u) \leq c\}$.

If the set of the critical points of $\Phi$, denoted by $\mathcal{K}:=\left\{u \in H: \Phi^{\prime}(u)=0\right\}$, is finite and $a<\inf \Phi(\mathcal{K})$, the critical groups of $\Phi$ at infinity are defined by (see [14])

$$
C_{q}(\Phi, \infty):=H_{q}\left(H, \Phi^{a}\right), \quad q \in \mathbf{N} \cup\{0\}
$$

For $q \in \mathbf{N} \cup\{0\}$, we call $\beta_{q}:=\operatorname{dim} C_{q}(\Phi, \infty)$ the Betti numbers of $\Phi$ and define the Morsetype numbers of the pair $\left(H, \Phi^{a}\right)$ by

$$
M_{q}:=M_{q}\left(H, \Phi^{a}\right)=\sum_{u \in \mathcal{K}} \operatorname{dim} C_{q}(\Phi, u) .
$$

With the above notations, we have the following facts (2.a)-(2.f) [15, Chapter 8].

(2.a) If $C_{\mu}(\Phi, \infty) \nsucceq 0$ for some $\mu \in \mathbf{N} \cup\{0\}$, then there exists $x_{0} \in \mathcal{K}$ such that

$$
C_{\mu}\left(\Phi, x_{0}\right) \not 0 \text {; }
$$


(2.b) If $\mathcal{K}=\left\{x_{0}\right\}$, then $C_{q}(\Phi, \infty) \cong C_{q}\left(\Phi, x_{0}\right)$;

(2.c) $\sum_{j=0}^{\infty}(-1)^{j} M_{j}=\sum_{j=0}^{\infty}(-1)^{j} \beta_{j}$.

If $x_{0} \in \mathcal{K}$ and $\Phi^{\prime \prime}\left(x_{0}\right)$ is a Fredholm operator and the Morse index $\mu_{0}$ and nullity $v_{0}$ of $x_{0}$ are finite, then we have

(2.d) $C_{q}\left(\Phi, x_{0}\right) \cong 0$ for $q \notin \mathbf{Z}\left[\mu_{0}, \mu_{0}+v_{0}\right]$;

(2.e) If $C_{\mu_{0}}\left(\Phi, x_{0}\right) \varsubsetneqq 0$, then $C_{q}\left(\Phi, x_{0}\right) \cong \delta_{q, \mu_{0}} \mathbf{Z}$, and if $C_{\mu_{0}+v_{0}}\left(\Phi, x_{0}\right) \varsubsetneqq 0$, then

$$
C_{q}\left(\Phi, x_{0}\right) \cong \delta_{q, \mu_{0}+v_{0}} \mathbf{Z}
$$

(2.f) If $m:=\operatorname{dim} H<+\infty$, then $C_{q}\left(\Phi, x_{0}\right) \cong \delta_{q, 0} \mathbf{Z}$ when $x_{0}$ is local minimizer of $\Phi$, while $C_{q}\left(\Phi, x_{0}\right) \cong \delta_{q, m} \mathbf{Z}$ when $x_{0}$ is the local maximizer of $\Phi$.

We say that $\Phi$ has a local linking at $x_{0} \in \mathcal{K}$ if there exists the direct sum decomposition: $H=H^{+} \oplus H^{-}$and $\epsilon>0$ such that

$$
\begin{aligned}
& \Phi(x)>\Phi\left(x_{0}\right) \quad \text { if } x-x_{0} \in H^{+}, 0<\left\|x-x_{0}\right\| \leq \epsilon, \\
& \Phi(x) \leq \Phi\left(x_{0}\right) \quad \text { if } x-x_{0} \in H^{-},\left\|x-x_{0}\right\| \leq \epsilon .
\end{aligned}
$$

The following results are due to $\mathrm{Su}[13]$.

(2.g) Assume that $\Phi$ has a local linking at $x_{0} \in \mathcal{K}$ with respect to $H=H^{+} \oplus H^{-}$and

$$
k=\operatorname{dim} H^{-}<+\infty \text {. Then }
$$

$$
\begin{aligned}
& C_{q}\left(\Phi, x_{0}\right) \cong \delta_{q, \mu_{0}} \mathbf{Z} \quad \text { if } k=\mu_{0}, \\
& C_{q}\left(\Phi, x_{0}\right) \cong \delta_{q, \mu_{0}+\nu_{0}} \mathbf{Z} \quad \text { if } k=\mu_{0}+v_{0} .
\end{aligned}
$$

We say that $\Phi$ satisfies the Cerami condition $((C)$ in short $)$ if every sequence $\left\{x_{n}\right\} \subset$ $H$ such that $\left\{\Phi\left(x_{n}\right)\right\}$ is bounded and $\left(1+\left\|x_{n}\right\|\right) \Phi^{\prime}\left(x_{n}\right) \rightarrow 0$ as $n \rightarrow \infty$ has a convergent subsequence. The following lemma derives from [11, Proposition 3.2].

Lemma 2.1 [11] Let $H$ be a Hilbert space, and $\left\{\Phi_{s} \in C^{1}(H, \mathbf{R}) \mid s \in[0,1]\right\}$ are a family of functionals such that $\Phi_{s}^{\prime}$ and $\partial_{s} \Phi_{s}$ are locally Lipschitz continuous. Assume that $\Phi_{0}$ and $\Phi_{1}$ satisfy $(C)$. If there exists $M>0$ such that

$$
\inf _{s \in[0,1],\|x\|>M}(1+\|x\|)\left\|\Phi_{s}^{\prime}(x)\right\|>0 \text { and } \inf _{s \in[0,1],\|x\| \leq M} \Phi_{s}(x)>-\infty
$$

then

$$
C_{q}\left(\Phi_{0}, \infty\right)=C_{q}\left(\Phi_{1}, \infty\right)
$$

Remark 2.1 The deformation lemma can be proved with the weaker condition (C) replacing the usual (PS) condition [16]. Therefore, if the (PS) condition is replaced by the (C) condition, (2.a)-(2.g) stated above still hold.

\section{Compactness and critical group at infinity}

In this section, we are going to prove the compactness of the associated energy functionals and to calculate the critical groups at infinity. First of all, let us introduce the variational structure for problem (BP). 


\subsection{Variational structure}

The class $E$ of functions $x: \mathbf{Z}[0, N+1] \mapsto R$ such that $x(0)=x(N+1)=0$, equipped with the inner product $\langle\cdot, \cdot\rangle$ and norm $\|\cdot\|$ as follows:

$$
\langle x, y\rangle=\sum_{k=1}^{N} x(k) y(k), \quad\|x\|=\left(\sum_{k=1}^{N}|x(k)|^{2}\right)^{1 / 2} \quad \text { for } x, y \in E,
$$

is linearly homeomorphic to $\mathbf{R}^{N}$. Denote $\theta=(0,0, \ldots, 0)^{T} \in \mathbf{R}^{N}$. Throughout this paper, we always identify $x \in E$ with $x=(x(1), x(2), \ldots, x(N))^{T} \in \mathbf{R}^{N}$.

$$
\begin{aligned}
\text { Set } \mathbf{f}(x) & =(f(1, x(1)), \ldots, f(N, x(N)))^{T}, x \in E \text { and } \\
A & =\left(\begin{array}{ccccccc}
2 & -1 & 0 & \cdots & 0 & 0 & 0 \\
-1 & 2 & -1 & \cdots & 0 & 0 & 0 \\
& \cdots & \cdots & \cdots & & \cdots & \\
0 & 0 & 0 & \cdots & -1 & 2 & -1 \\
0 & 0 & 0 & \cdots & 0 & -1 & 2
\end{array}\right)_{N \times N} .
\end{aligned}
$$

Then we can equivalently rewrite (BP) as a nonlinear algebraic system

$$
A x=\mathbf{f}(x), \quad x \in E .
$$

Denote $E^{l}=\operatorname{ker}\left(A-\lambda_{l} I\right), l=1, \ldots, N$, where $I$ is the identity operator. Thus $\operatorname{dim} E^{l}=1$, $l=1,2, \ldots, N$. Set

$$
E^{-}=\bigoplus_{l=1}^{h-1} E^{l}, \quad E^{+}=\left(\bigoplus_{l=1}^{h+1} E^{l}\right)^{\perp}, \quad E^{v}=E^{-} \oplus E^{+},
$$

then $E$ has the decomposition $E=E^{h} \oplus E^{h+1} \oplus E^{v}$. In the rest of this paper, the expression $x=x^{h}+x^{h+1}+x^{v}$ for $x \in E$ always means $x^{\dagger} \in E^{\dagger}, \dagger=h, h+1, v$.

Define a functional $J: E \rightarrow \mathbf{R}$ by

$$
J(x)=\frac{1}{2}\langle A x, x\rangle-\sum_{k=1}^{N} F(k, x(k)) \quad \text { for } x \in E,
$$

where $F(k, t)=\int_{0}^{t} f(k, s) d s,(k, t) \in \mathbf{Z}[1, N] \times \mathbf{R}$. Then the Fréchet derivative of $J$ at $x \in E$, denoted by $J^{\prime}(x)$, can be described by (see [3])

$$
\left\langle J^{\prime}(x), y\right\rangle=\langle A x, y\rangle-\sum_{k=1}^{N} f(k, x(k)) y(k) \quad \text { for } y \in E .
$$

Remark 3.1 From (3.2) we see that $x \in E$ is a critical point of $J$ if and only if $x$ is a solution of (3.1) (or equivalently (BP)). In addition, $J$ is $C^{2}$-differentiable with

$$
\left\langle J^{\prime \prime}(x) y, z\right\rangle=\langle A y, z\rangle-\sum_{k=1}^{N} f^{\prime}(k, x(k)) y(k) z(k) \quad \text { for } y, z \in E .
$$




\subsection{Compactness of related functionals}

Define a family of functionals $J_{s}: E \rightarrow \mathbf{R}, s \in[0,1]$ by

$$
J_{s}(x)=\frac{1}{2}\langle A x, x\rangle-\frac{1-s}{4}\left(\lambda_{h}+\lambda_{h+1}\right)\|x\|^{2}-s \sum_{k=1}^{N} F(k, x(k)) \quad \text { for } x \in E \text {, }
$$

then the Fréchet derivative of $J_{s}$ at $x \in E$, denoted by $J_{s}^{\prime}(x)$, can be described by (see [3])

$$
\left\langle J_{s}^{\prime}(x), y\right\rangle=\langle A x, y\rangle-\sum_{k=1}^{N} f_{s}(k, x(k)) y(k) \quad \text { for } y \in E,
$$

where $s \in[0,1]$ and

$$
f_{s}(k, t)=s f(k, t)+\frac{1-s}{2}\left(\lambda_{h}+\lambda_{h+1}\right) t \quad \text { for }(k, t) \in \mathbf{Z}[1, N] \times \mathbf{R} .
$$

Lemma 3.1 Assume that $\left(\mathrm{f}_{\infty}\right)$ holds. For any sequences $\left\{x_{n}\right\} \subset E$ and $\left\{s_{n}\right\} \subset[0,1],\left\{x_{n}\right\}$ is bounded provided that

$$
\left(1+\left\|x_{n}\right\|\right) J_{s_{n}}^{\prime}\left(x_{n}\right) \rightarrow 0 \text { as } n \rightarrow \infty
$$

Moreover, for every $\hat{s} \in[0,1], J_{\hat{s}}$ satisfies $(C)$.

Proof Assume, for a contradiction, that $\left\{x_{n}\right\}$ is unbounded. Then there exists a subsequence, which we still call $\left\{x_{n}\right\}$, with $K \subset \mathbf{Z}[1, N]$ being nonempty such that

$$
\lim _{n \rightarrow \infty} x_{n}(k)=\infty \quad \text { for } k \in K
$$

and either $K=\mathbf{Z}[1, N]$ or, for any fixed $k \in K^{c} \equiv \mathbf{Z}[1, N] \backslash K,\left\{x_{n}(k)\right\}$ is a bounded sequence.

Noticing that [10, Lemma 3.7], with its proof being modified slightly, is applicable here, we know that

$$
\text { either } \frac{\left\|x_{n}^{h}\right\|}{\left\|x_{n}\right\|} \rightarrow 1 \quad \text { or } \quad \frac{\left\|x_{n}^{h+1}\right\|}{\left\|x_{n}\right\|} \rightarrow 1 \quad \text { as } n \rightarrow \infty
$$

Set

$$
\begin{aligned}
& \Gamma_{1}:=\limsup _{n \rightarrow \infty} \sum_{k=1}^{N}\left\{f_{s_{n}}\left(k, x_{n}(k)\right)-\lambda_{h} x_{n}(k)\right\} x_{n}^{h}(k), \\
& \Gamma_{2}:=\liminf _{n \rightarrow \infty} \sum_{k=1}^{N}\left\{f_{s_{n}}\left(k, x_{n}(k)\right)-\lambda_{h+1} x_{n}(k)\right\} x_{n}^{h+1}(k) .
\end{aligned}
$$

Thus we have two cases to be considered.

Case 1. $\left\|x_{n}^{h}\right\| /\left\|x_{n}\right\| \rightarrow 1$ as $n \rightarrow \infty$. We have $\left\|x_{n}^{h}\right\| \rightarrow \infty$ as $n \rightarrow \infty$ and

$$
\lim _{n \rightarrow \infty} \frac{\left\|x_{n}^{h+1}\right\|}{\left\|x_{n}\right\|}=0, \quad \lim _{n \rightarrow \infty} \frac{\left\|x_{n}^{v}\right\|}{\left\|x_{n}\right\|}=0
$$


By $\left(\mathrm{f}_{\infty}\right)(\mathrm{i})$, there exist $M>0$ and $\xi>0$ such that $t^{2}\left(f(k, t) / t-\lambda_{h}\right)>\xi$ and $t^{2}\left(\lambda_{h+1}-\lambda_{h}\right)>\xi$ for $|t|>M$ and $k \in \mathbf{Z}[1, N]$. Then, for $|t|>M, k \in \mathbf{Z}[1, N]$ and $s \in[0,1]$,

$$
\begin{aligned}
\left(\frac{f_{s}(k, t)}{t}-\lambda_{h}\right) t^{2} & =s\left(\frac{f(k, t)}{t}-\lambda_{h}\right) t^{2}+\frac{1-s}{2}\left(\lambda_{h+1}-\lambda_{h}\right) t^{2} \\
& \geq s \xi+\frac{1-s}{2} \xi \geq \frac{\xi}{2} .
\end{aligned}
$$

Choose $N_{1}>0$ such that $\left|x_{n}(k)\right|>M$ for $k \in K$ and $n>N_{1}$. It follows that

$$
\begin{aligned}
& \left\{f_{s_{n}}\left(k, x_{n}(k)\right)-\lambda_{h} x_{n}(k)\right\} x_{n}^{h}(k) \\
& \quad=\left\{\frac{f_{s_{n}}\left(k, x_{n}(k)\right)}{x_{n}(k)}-\lambda_{h}\right\}\left(x_{n}(k)\right)^{2}\left(\frac{\left(x_{n}(k)-z_{n}(k)\right)}{x_{n}(k)}\right) \\
& \quad \geq\left\{\frac{f_{s_{n}}\left(k, x_{n}(k)\right)}{x_{n}(k)}-\lambda_{h}\right\}\left(x_{n}(k)\right)^{2}\left(\frac{\left(\left|x_{n}(k)\right|-\left|z_{n}(k)\right|\right)}{\left\|x_{n}\right\|}\right) \\
& \quad \geq \frac{\xi\left(\left|x_{n}(k)\right|-\left|z_{n}(k)\right|\right)}{2\left\|x_{n}\right\|} \quad \text { for } k \in K \text { and } n>N_{1},
\end{aligned}
$$

where $z_{n}=x_{n}^{h+1}+x_{n}^{\nu}$. Since $E$ possesses an equivalent norm defined by $\|x\|_{1} \equiv \sum_{k=1}^{N}|x(k)|$ for $x \in E$, there exists a positive constant $C>0$ such that $\|x\|_{1} \geq C\|x\|, x \in E$. Thus, by (3.6) and (3.7),

$$
\begin{aligned}
\Gamma_{1} & \geq \limsup _{n \rightarrow \infty} \frac{\xi}{2\left\|x_{n}\right\|}\left\{\sum_{k \in K}\left|x_{n}(k)\right|-\sum_{k \in K}\left|z_{n}(k)\right|\right\} \\
& =\limsup _{n \rightarrow \infty} \frac{\xi}{2\left\|x_{n}\right\|}\left\{\sum_{k=1}^{N}\left|x_{n}(k)\right|-\sum_{k=1}^{N}\left|z_{n}(k)\right|\right\} \\
& \geq \limsup _{n \rightarrow \infty} \frac{\xi}{2\left\|x_{n}\right\|}\left(C\left\|x_{n}\right\|-\sqrt{p}\left\|z_{n}\right\|\right)=\frac{C \xi}{2},
\end{aligned}
$$

where the equality holds because $\left|x_{n}(k)\right| /\left\|x_{n}\right\| \rightarrow 0$ as $n \rightarrow \infty$ for $k \in K^{c}$ in case $K^{c} \neq \varnothing$.

Case 2. $\left\|x_{n}^{h+1}\right\| /\left\|x_{n}\right\| \rightarrow 1$ as $n \rightarrow \infty$. In this case, by using $\left(\mathrm{f}_{\infty}\right)$ (ii), we can show that $\Gamma_{2}<0$ in the same way.

On the other hand, it follows from (3.5) that

$$
\left\langle\left\|x_{n}\right\| \cdot J_{s_{n}}^{\prime}\left(x_{n}\right), \frac{x_{n}^{\dagger}}{\left\|x_{n}^{\dagger}\right\|}\right\rangle \rightarrow 0 \quad \text { as } n \rightarrow \infty, \dagger=h, h+1,
$$

which implies that

$$
\left\langle J_{s_{n}}^{\prime}\left(x_{n}\right), x_{n}^{\dagger}\right\rangle \rightarrow 0 \quad \text { as } n \rightarrow \infty, \dagger=h, h+1
$$

that is,

$$
\left\langle A x_{n}, x_{n}^{\dagger}\right\rangle-\sum_{k=1}^{N} f_{s_{n}}\left(k, x_{n}(k)\right) x_{n}^{\dagger}(k) \rightarrow 0 \quad \text { as } n \rightarrow \infty, \dagger=h, h+1 .
$$

Note that $\left\langle A x_{n}, x_{n}^{\dagger}\right\rangle=\left\langle\lambda_{\dagger} x_{n}, x_{n}^{\dagger}\right\rangle, \dagger=h, h+1$, it follows that $\Gamma_{1}=\Gamma_{2}=0$. This contradiction proves the first conclusion. 
By setting $s_{n} \equiv \hat{s} \in[0,1]$ in the proven conclusion, we see that $J_{\hat{s}}$ satisfies $(C)$. The proof is complete.

For $x \in E$, set $x^{+}(k)=\max \{0, x(k)\}, k \in \mathbf{Z}[1, N]$ and $x^{+}=\left(x^{+}(1), \ldots, x^{+}(N)\right)^{T}$. The following lemma is derived from [3, Lemma 2.1].

Lemma 3.2 [3] If $x$ is a solution of

$$
A x=\mathbf{f}\left(x^{+}\right), \quad x \in E,
$$

then $x \geq \theta$ and hence it is also a solution of (3.1). Moreover, either $x>\theta$ or $x=\theta$.

For $x, y \in E$, we say that $x \geq y(x>y)$ if $x(k) \geq y(k)(x(k)>y(k))$ for $k \in \mathbf{Z}[1, N]$.

Lemma 3.3 Let $\varsigma_{j}$ be the eigenvector corresponding to $\lambda_{j}, j \in \mathbf{Z}[1, N]$, then $\varsigma_{1}$ can be chosen to satisfy $\varsigma_{1}>0$. Moreover, for $j \geq 2$, neither $\varsigma_{j} \geq \theta$ nor $\varsigma_{j} \leq \theta$.

Proof First we claim that $\varsigma_{1} \geq \theta$ or $\varsigma_{1} \leq \theta$. Otherwise, by setting $\bar{\zeta}_{1}=\left(\left|\varsigma_{1}(1)\right|, \ldots,\left|\varsigma_{1}(N)\right|\right)^{T}$, we have

$$
\sum_{k=1}^{N}\left|\Delta \bar{\zeta}_{1}(k)\right|^{2}<\sum_{k=1}^{N}\left|\Delta \varsigma_{1}(k)\right|^{2}
$$

Since $\lambda_{1}=\inf _{\|x\|=1}\langle A x, x\rangle=\left\langle A \varsigma_{1}, \varsigma_{1}\right\rangle /\left\|\varsigma_{1}\right\|^{2}$, it follows from (3.8) that

$$
\begin{aligned}
\lambda_{1} & \leq \frac{\left\langle A \bar{\zeta}_{1}, \bar{\zeta}_{1}\right\rangle}{\left\|\bar{\zeta}_{1}\right\|}=\frac{\sum_{k=1}^{N}\left|\Delta \bar{\zeta}_{1}(k)\right|^{2}}{\left\|\varsigma_{1}\right\|^{2}} \\
& <\frac{\sum_{k=1}^{N}\left|\Delta \zeta_{1}(k)\right|^{2}}{\left\|\zeta_{1}\right\|^{2}}=\lambda_{1} .
\end{aligned}
$$

This contradiction proves the above claim. Thus $\varsigma_{1}$ can be assumed to satisfy $\varsigma_{1} \geq \theta$ and then $A \varsigma_{1}=\lambda_{1} \varsigma_{1}^{+}$. It follows by Lemma 3.2 that $\varsigma_{1}>\theta$ and the first conclusion holds. Further, for $k \geq 2, \varsigma_{k}$ and $\varsigma_{1}$ are orthogonal to each other, which implies that neither $\varsigma_{j} \geq \theta$ nor $\varsigma_{j} \leq \theta$. The proof is complete.

Lemma 3.4 Let the function $g \in C(\mathbf{Z}[1, N] \times \mathbf{R}, \mathbf{R})$ be such that $g(k, t)=0$ for $t<0$. Assume that there exists $h \in \mathbf{Z}[2, N]$ such that

$$
\lambda_{h} \leq \liminf _{t \rightarrow+\infty} \frac{g(k, t)}{t} \leq \limsup _{t \rightarrow+\infty} \frac{g(k, t)}{t} \leq \lambda_{h+1} .
$$

Then the functional

$$
I(x)=\frac{1}{2}\langle A x, x\rangle-\sum_{k=1}^{N} G(k, x(k))
$$

satisfies the $(P S)$ condition, where $G(k, t)=\int_{0}^{t} g(k, s) d s$. 
Proof Let $\left\{x_{n}\right\} \subset E$ be such that

$$
I^{\prime}\left(x_{n}\right) \rightarrow 0 \quad \text { as } n \rightarrow \infty
$$

We only need to prove that $\left\{x_{n}\right\}$ is bounded. In fact, if $\left\{x_{n}\right\}$ is unbounded, there exists a subsequence, still called $\left\{x_{n}\right\}$, such that $\left\|x_{n}\right\| \rightarrow \infty$ as $n \rightarrow \infty$.

Let $w_{n}=x_{n} /\left\|x_{n}\right\|$, then $\left\|w_{n}\right\|=1$. There is a convergent subsequence of $\left\{w_{n}\right\}$, call it $\left\{w_{n}\right\}$ again, such that $w_{n} \rightarrow w \in E$ as $n \rightarrow \infty$. For every $y \in E$, we have $\left\langle I^{\prime}\left(x_{n}\right), y\right\rangle /\left\|x_{n}\right\| \rightarrow 0$ as $n \rightarrow \infty$, that is,

$$
\left\langle A w_{n}, y\right\rangle-\sum_{k=1}^{N} \frac{g\left(k, x_{n}(k)\right)}{\left\|x_{n}\right\|} y(k) \rightarrow 0 \quad \text { as } n \rightarrow \infty
$$

Set

$$
K_{+}=\left\{k \in \mathbf{Z}[1, N] \mid x_{n}(k) \rightarrow+\infty \text { as } n \rightarrow \infty\right\} .
$$

We claim that $K_{+} \neq \varnothing$, since otherwise (3.11) leads to $\left\langle A w_{n}, y\right\rangle \rightarrow 0(n \rightarrow \infty)$ for $y \in E$, which leads to $w=0$, a contradiction. Thus we have by (3.9) that

$$
\lambda_{h} \leq \liminf _{n \rightarrow \infty} \frac{g\left(k, x_{n}(k)\right)}{x_{n}(k)} \leq \limsup _{n \rightarrow \infty} \frac{g\left(k, x_{n}(k)\right)}{x_{n}(k)} \leq \lambda_{h+1} \quad \text { for } k \in K_{+} \text {, }
$$

which implies that there exists a subsequence of $\left\{x_{n}\right\}$, still called $\left\{x_{n}\right\}$, and $\alpha_{k} \in\left[\lambda_{h}, \lambda_{h+1}\right]$, $k \in K_{+}$, such that

$$
\lim _{n \rightarrow \infty} \frac{g\left(k, x_{n}(k)\right)}{x_{n}(k)}=\alpha_{k} \quad \text { for } k \in K_{+} \text {. }
$$

If $k \in \mathbf{Z}[1, N] \backslash K_{+}$, then $g\left(k, x_{n}(k)\right) /\left\|x_{n}\right\| \rightarrow 0$ as $n \rightarrow \infty$. Thus we can rewrite (3.11) as

$$
\left\langle A w_{n}, y\right\rangle-\sum_{k \in K_{+}} \frac{g\left(k, x_{n}(k)\right)}{x_{n}(k)} \frac{x_{n}(k)}{\left\|x_{n}\right\|} y(k) \rightarrow 0 \quad \text { as } n \rightarrow \infty
$$

Letting $n \rightarrow \infty$ in (3.13) and using (3.12), we get

$$
\langle A w, y\rangle=\sum_{k \in K_{+}} \alpha_{k} w(k) y(k) \quad \text { for } y \in E
$$

Since $w(k) \geq 0$ for $k \in K_{+}$, it follows from (3.14) that

$$
A w=\sum_{k \in k_{+}} \alpha_{k} w^{+}(k)
$$

which, by Lemma 3.2, implies that $w>0$ and hence $K_{+}=\mathbf{Z}[1, N]$. Thus, (3.14) can be rewritten as

$$
\langle A w, y\rangle=\sum_{k=1}^{N} \alpha_{k} w(k) y(k) \quad \text { for } y \in E .
$$


Noticing that [10, Lemma 3.4], with its proof being modified slightly, is applicable here, we know that $w$ is an eigenvector corresponding to $\lambda_{h}$ or $\lambda_{h+1}$. Since $h \geq 2$, it follows from Lemma 3.3 that $w \ngtr \theta$. This contradiction completes the proof.

\subsection{Critical group at infinity}

Lemma 3.5 Letf satisfy $\left(\mathrm{f}_{\infty}\right)$. Then

$$
C_{q}(J, \infty) \cong \delta_{q, h} \mathbf{Z}, \quad C_{q}(-J, \infty) \cong \delta_{q, N-h} \mathbf{Z} .
$$

Proof We claim that there exists $M>0$ such that

$$
\inf \left\{(1+\|x\|)\left\|J_{s}^{\prime}(x)\right\|:\|x\|>M, s \in[0,1]\right\}>0,
$$

otherwise there exist $\left\{x_{n}\right\} \subset E$ and $\left\{s_{n}\right\} \subset[0,1]$ such that $\left\|x_{n}\right\| \rightarrow \infty$ and $\left(1+\left\|x_{n}\right\|\right) J\left(x_{n}\right) \rightarrow$ 0 as $n \rightarrow \infty$, which contradict Lemma 3.1. Moreover, it is easy to see that $\inf \left\{J_{s}(x): s \in\right.$ $[0,1],\|x\| \leq M\}>-\infty$. Thus, by Lemma 2.1 , we have

$$
C_{q}(J, \infty) \cong C_{q}\left(J_{0}, \infty\right)
$$

On the other hand,

$$
J_{0}(x)=\frac{1}{2}\langle A x, x\rangle-\frac{1}{4}\left(\lambda_{h}+\lambda_{h+1}\right)\|x\|^{2} .
$$

Note that $x=\theta$ is the unique critical point of $J_{0}$ with the Morse index $\mu:=\operatorname{dim}\left(E^{-} \oplus E^{h}\right)=h$ and nullity $v=0$. Then, by (2.b) and (2.e),

$$
C_{q}\left(J_{0}, \infty\right) \cong C_{q}\left(J_{0}, 0\right) \cong \delta_{q, h} \mathbf{Z}
$$

Similarly, we have $C_{q}(-J, \infty) \cong C_{q}\left(-J_{0}, 0\right) \cong \delta_{q, N-h} \mathbf{Z}$. The proof is complete.

\section{Proofs of main results}

Now we prove the main results of this paper. First, by applying (3.16) and (2.a), we know that $J$ has a critical point $x^{*}$ satisfying

$$
C_{h}\left(J, x^{*}\right) \neq 0
$$

Define $\alpha_{k}=f^{\prime}\left(k, x^{*}(k)\right), k \in \mathbf{Z}[1, N]$. Then from (3.3) we know by calculation that $\operatorname{ker} J_{1}^{\prime \prime}\left(x^{*}\right)$ is the solution space of the system $B x=0, x \in E$, where

$$
B=\left(\begin{array}{ccccccc}
2-\alpha_{1} & -1 & 0 & \cdots & 0 & 0 & 0 \\
-1 & 2-\alpha_{2} & -1 & \cdots & 0 & 0 & 0 \\
& \cdots & \cdots & \cdots & & \cdots & \\
0 & 0 & 0 & \cdots & -1 & 2-\alpha_{N-1} & -1 \\
0 & 0 & 0 & \cdots & 0 & -1 & 2-\alpha_{N}
\end{array}\right)_{N \times N}
$$


Thus $v_{1}=\operatorname{dim} \operatorname{ker} J_{1}^{\prime \prime}\left(x^{*}\right) \leq 1$ since $B$ possesses non-degenerate $(N-1)$ order submatrixes. By (2.d)-(2.e), we further have

$$
C_{q}\left(J, x^{*}\right) \cong \delta_{q, h} \mathbf{Z}
$$

Proof of Theorem 1.1 First we give the proof for the case (i). By $f^{\prime}(k, 0)<\lambda_{1}$ for $k \in \mathbf{Z}[1, N]$, we know that $x=\theta$ is a strict local minimizer of $J$. Thus, by (2.f), we have correspondingly

$$
C_{q}(J, \theta) \cong \delta_{q, 0} \mathbf{Z}
$$

Noticing that $h \geq 2$, by comparing (4.2) with (4.1), we have $x^{*} \neq \theta$.

For $k \in \mathbf{Z}[1, N]$, set $f^{+}(k, t)=f(k, t)$ for $t \geq 0, f^{+}(k, t)=0$ for $t<0$. Let $F^{+}(k, t)=$ $\int_{0}^{t} f^{+}(k, s) d s$. Then the critical points of

$$
J^{+}(x)=\frac{1}{2}\langle A x, x\rangle-\sum_{k=1}^{N} F^{+}(k, x(k))
$$

are exactly solutions of the problem

$$
A x=\mathbf{f}^{+}\left(x^{+}\right)
$$

where $\mathbf{f}^{+}(x)=\left(f^{+}(1, x(1)), \ldots, f^{+}(N, x(N))\right)^{T}, x \in E$. By Lemma 3.4, we see that $J^{+} \in$ $C^{2-0}(E, \mathbf{R})$ satisfies the (PS) condition. From the definition of $f^{+}(k, \cdot)$ and the assumption $f^{\prime}(k, 0)<\lambda_{1}, k \in \mathbf{Z}[1, N]$, we know that there exists $\eta>0$ such that $\left(\lambda_{1} t-f^{+}(k, t)\right) t>0$ for $t \in(-\eta, \eta) \backslash\{0\}$. For any fixed $x \in E$ with $0<\|x\|<\eta$, define a function $\phi(s)=J^{+}(s x)$, $s \in[0,1]$. By the Lagrange mean value theorem, there exists $\xi \in(0,1)$ such that

$$
\begin{aligned}
J^{+}(x) & =\phi(1)-\phi(0)=\phi^{\prime}(\xi) \\
& =\langle A(\xi x), x\rangle-\sum_{k=1}^{N} f^{+}(k, \xi x(k)) x(k) \\
& \geq \sum_{k=1}^{N}\left\{\lambda_{1} \xi x(k)-f^{+}(k, \xi x(k))\right\} x(k)>0,
\end{aligned}
$$

which implies that there exist $\rho>0$ and $\tau>0$ such that

$$
J^{+}(x) \geq \tau, \quad x \in E \text { with }\|x\|=\rho .
$$

In addition, let $\varsigma_{1}$ be the eigenvector of $A$ corresponding to $\lambda_{1}$ with $\varsigma_{1}>0$, then $\left(f_{\infty}\right)$, with $h \in \mathbf{Z}[2, N-1]$, implies that

$$
J^{+}\left(t \varsigma_{1}\right) \rightarrow-\infty \quad \text { as } t \rightarrow+\infty .
$$

By Mountain Pass Theorem [17, 18], $J^{+}$has a critical point $x_{1} \neq \theta$ with the critical group property for a mountain pass point [17], that is, $C_{1}\left(J^{+}, x_{1}\right) \varsubsetneqq 0$. Noticing that $x_{1}$ satisfies 
(4.3), we get by Lemma 3.2 that $x_{1}>\theta$ and hence $x_{1}$ is also a mountain pass point of $J$, that is, $C_{1}\left(J, x_{1}\right) \supsetneqq 0$.

The same argument shows that $J$ has a nontrivial critical point $x_{2}<\theta$ with $C_{1}\left(J, x_{2}\right) \varsubsetneqq 0$. Noticing that $h \geq 2$, by comparing the critical groups, we see that $x_{1}, x_{2}$ and $x^{*}$ are three nontrivial critical points of $J$.

By the same argument as that for (4.1), we get $C_{q}\left(J, x_{i}\right) \cong \delta_{q, 1} \mathbf{Z}, i=1,2$. If $x_{1}, x_{2}$ and $x^{*}$ are all the nontrivial critical points of $J$, then $\mathcal{K}=\left\{\theta, x_{1}, x_{2}, x^{*}\right\}$ and then (2.c) reads

$$
(-1)^{0} \times 1+(-1)^{1} \times 2+(-1)^{h} \times 1=(-1)^{h} \times 1,
$$

a contradiction. Thus we claim that there exist at least four nontrivial critical points of $J$.

In the case (ii), we consider the functional -J. By applying (3.16) and (2.a), we know that $-J$ possesses a critical point $x_{1}^{*}$ satisfying

$$
C_{N-h}\left(-J, x_{1}^{*}\right) \neq 0
$$

Since $f^{\prime}(k, 0)>\lambda_{N}$ for $k \in \mathbf{Z}[1, N], x=\theta$ is a strict local minimizer of $-J$ and

$$
C_{q}(-J, \theta) \cong \delta_{q, 0} \mathbf{Z}
$$

Noticing that $h \leq N-2$, we know by comparing (4.5) with (4.4) that $x_{1}^{*} \neq \theta$. The rest of the arguments are similar to that in case (i) and will be omitted. The proof is complete.

Proof of Theorem 1.2 In view of (3.3) and the assumption $\lambda_{m}<f^{\prime}(k, 0)<\lambda_{m+1}, k \in \mathbf{Z}[1, N]$, we see that $x=\theta$ is a non-degenerate critical point of $J$ with the Morse index $\mu_{0}=m$. Thus

$$
C_{q}(J, 0) \cong \delta_{q, m} \mathbf{Z}
$$

Noticing that $h \neq m$, we know by comparing (4.6) with (4.1) that $x^{*} \neq \theta$.

We may assume that $t_{0}>0$ in $\left(\mathrm{f}_{0}\right)$. For $k \in \mathbf{Z}[1, N]$, set

$$
\tilde{f}(k, t)= \begin{cases}0, & t<0, \\ f(k, t), & t \in\left[0, t_{0}\right], \\ 0, & t>t_{0} .\end{cases}
$$

Define

$$
\tilde{J}(x)=\frac{1}{2}\langle A x, x\rangle-\sum_{k=1}^{N} \tilde{F}(k, x(k)), \quad x \in E,
$$

where $\tilde{F}(k, t)=\int_{0}^{t} \tilde{f}(k, s) d s$. Since $\tilde{J}(x) \rightarrow+\infty$ as $\|x\| \rightarrow \infty$, there is a minimizer $x_{0}$ of $\tilde{J}$. Thus

$$
A x_{0}=\tilde{\mathbf{f}}\left(x_{0}\right),
$$


where $\tilde{\mathbf{f}}(x)=(\tilde{f}(1, x(1)), \tilde{f}(2, x(2)), \ldots, \tilde{f}(N, x(N)))^{T}$ for $x \in E$. By the definition of $\tilde{f}$, the above equality can be rewritten as

$$
A x_{0}=\tilde{\mathbf{f}}\left(x_{0}^{+}\right) .
$$

From Lemma 3.4, we know that $x_{0}=\theta$ or $x_{0}>\theta$. By assumption $f^{\prime}(k, 0) \in\left(\lambda_{m}, \lambda_{m+1}\right)$ and $m \geq 2$, we know that $\theta$ is not a minimizer. Thus we have $x_{0}>\theta$. In the same way as the proof of Lemma 3.2, we can prove that $x_{0}(k)<t_{0}$ for $k \in \mathbf{Z}[1, N]$. Thus $x_{0}$ is a local minimizer of $J$, therefore

$$
C_{q}\left(J, x_{0}\right) \cong \delta_{q, 0} \mathbf{Z}
$$

Define $\hat{f}(k, t)=f\left(k, t+x_{0}(k)\right)-f\left(k, x_{0}(k)\right),(k, t) \in \mathbf{Z}[1, N] \times \mathbf{R}$ and consider the functional

$$
\hat{J}(z)=\frac{1}{2}\langle A z, z\rangle-\sum_{k=1}^{N} \hat{F}(k, z(k)), \quad z \in E,
$$

where $\hat{F}(k, t)=\int_{0}^{t} \hat{f}(k, s) d s$. A simple calculation shows that if $z$ is a positive critical point of $\hat{J}$, then $x_{0}+z$ is a critical point of $J$, and, moreover, $C_{q}(\hat{J}, z)=C_{q}\left(J, x_{0}+z\right)$.

Furthermore, define

$$
\hat{f}^{+}(k, t)=\left\{\begin{array}{ll}
\hat{f}(k, t), & t \geq 0, \\
0, & t<0,
\end{array} \quad k \in \mathbf{Z}[1, N]\right.
$$

and its energy functional

$$
\hat{J}^{+}(z)=\frac{1}{2}\langle A z, z\rangle-\sum_{k=1}^{N} \hat{F}^{+}(k, z(k)), \quad z \in E,
$$

where $\hat{F}^{+}(k, t)=\int_{0}^{t} \hat{f}^{+}(k, s) d s$. By $\left(\mathrm{f}_{\infty}\right)$, we see that $\hat{f}^{+}$satisfies

$$
\lambda_{h} \leq \liminf _{t \rightarrow+\infty} \frac{\hat{f}^{+}(k, t)}{t} \leq \liminf _{t \rightarrow+\infty} \frac{\hat{f}^{+}(k, t)}{t} \leq \lambda_{h+1}, \quad k \in \mathbf{Z}[1, N] .
$$

It follows from Lemma 3.4 that $\hat{J}^{+}$satisfies the (PS) condition. If $x_{0}$ is not a strict local minimizer of $J$, then there exists infinitely many critical points near $x_{0}$ and the conclusion holds. Now we assume that $x_{0}$ is a strict local minimizer of $J$, then $z=\theta$ is a strict local minimizer of $\hat{J}^{+}$. In the same way as the proof of Theorem 1.1, we know that $\hat{J}^{+}$has a critical point $z_{1}$, which is a mountain pass point of $\hat{J}^{+}$with $C_{1}\left(\hat{J}^{+}, z_{1}\right) \neq 0$ and $z_{1}>\theta$. Thus $z_{1}$ is also a critical point of $\hat{J}$ with $C_{1}\left(\hat{J}, z_{1}\right) \varsubsetneqq 0$. Hence $x_{1}=x_{0}+z_{1}$ is a critical point of $J$ with $C_{1}\left(J, x_{1}\right) \nsucceq 0$.

In a similar way, we know that $J$ has a critical point $x_{2}<x_{0}$ with $C_{1}\left(J, x_{2}\right) \supsetneqq 0$. Finally, by comparing the critical groups and by using the condition $m, h \geq 2$ with $m \neq k$, we see that $x^{*}, x_{0}, x_{1}$ and $x_{2}$ are four nontrivial critical points of $J$ in which $x_{0}$ and $x_{1}$ are positive. The proof is complete.

The proof of the following lemma is similar to that of [19, Theorem 3.1] and is omitted. 
Lemma 4.1 [19] Let $f$ satisfy $\left(\mathrm{F}_{0}^{+}\right)\left(\right.$or $\left.\left(\mathrm{F}_{0}^{-}\right)\right)$. Then $J$ has a local linking at $x=\theta$ with respect to the decomposition $E=H^{-} \oplus E^{+}$, where $E^{-}:=\bigoplus_{l \leq m} E^{l}$ (or $E^{-}:=\bigoplus_{l<m} E^{l}$ respectively).

Proof of Theorem 1.3 In view of (3.3) and the assumption $f^{\prime}(k, 0)=\lambda_{m}, k \in \mathbf{Z}[1, N]$, we see that $x=\theta$ is a degenerate critical point of $J$ with the Morse index $\mu_{0}=m-1$ and nullity $v_{0}=1$. By Lemma 4.1 and $(2 . \mathrm{g})$, we have, corresponding to $\left(\mathrm{F}_{0}^{-}\right)$or $\left(\mathrm{F}_{0}^{+}\right)$respectively,

$$
C_{q}(J, 0) \cong \delta_{q, m-1} \mathbf{Z} \quad \text { or } \quad C_{q}(J, 0) \cong \delta_{q, m} \mathbf{Z}
$$

which, compared with (4.1), implies that $x^{*} \neq 0$ in both of cases (i) and (ii). The rest of the proof is similar to that of Theorem 1.2 and will be omitted. The proof is complete.

\section{Competing interests}

The author declares that they have no competing interests.

\section{Acknowledgements}

The author is grateful for the referees' careful reviewing and helpful comments. This work is supported by Beijing Municipal Commission of Education (KZ201310028031, KM2014).

Received: 15 May 2013 Accepted: 14 October 2013 Published: 08 Nov 2013

\section{References}

1. Agarwal, RP: Difference Equations and Inequalities. Monographs and Textbook in Pure and Applied Mathematics, vol. 228. Dekker, New York (2000)

2. Elaydi, S: An Introduction to Difference Equations. Springer, New York (2005)

3. Agarwal, RP, Perera, K, O'Regan, D: Multiple positive solutions of singular and nonsingular discrete problems via variational methods. Nonlinear Anal. 58, 69-73 (2004)

4. Bonanno, G, Candito, P: Nonlinear difference equations investigated via critical point methods. Nonlinear Anal. 70 , 3180-3186 (2009)

5. Galewski, M, Smejda, J: On variational methods for nonlinear difference equations. J. Comput. Appl. Math. 233 , 2985-2993 (2010)

6. Liu, JS, Wang, SL, Zhang, JM, Zhang, FW: Nontrivial solutions for discrete boundary value problems with multiple resonance via computations of the critical groups. Nonlinear Anal. TMA 75, 3809-3820 (2012)

7. Zhu, BS, Yu, JS: Multiple positive solutions for resonant difference equations. Math. Comput. Model. 49, 1928-1936 (2009)

8. Mawhin, J: Periodic solutions of second order nonlinear difference systems with $\phi$-Laplacian: a variational approach. Nonlinear Anal. TMA 75, 4672-4687 (2012)

9. Tan, FH, Guo, ZM: Periodic solutions for second-order difference equations with resonance at infinity. J. Differ. Equ. Appl. 18, 149-161 (2012)

10. Zhang, XS, Wang, D: Multiple periodic solutions for difference equations with double resonance at infinity. Adv. Differ. Equ. 2011, Article ID 806458 (2011). doi:10.1155/2011/806458

11. Liang, ZP, Su, JB: Multiple solutions for semilinear elliptic boundary value problems with double resonance. J. Math. Anal. Appl. 354, 147-158 (2009)

12. Robinson, S: Double resonance in semilinear elliptic boundary value problem over bounded and unbounded domains. Nonlinear Anal. TMA 21, 407-424 (1993)

13. Su, JB: Semilinear elliptic boundary value problems with double resonance between two consecutive eigenvalues. Nonlinear Anal. 48, 881-895 (2002)

14. Bartsch, T, Li, SJ: Critical point theory for asymptotically quadratic functionals and applications to problems with resonance. Nonlinear Anal. TMA 28, 419-441 (1997)

15. Mawhin, J, Willem, M: Critical Point Theory and Hamiltonian Systems. Springer, Berlin (1989)

16. Cerami, G: Un criterio di esistenza per i punti critici su varietâ illimitate. Rend. - Ist. Lomb., Accad. Sci. Lett., a Sci. Mat. Fis. Chim. Geol. 112, 332-336 (1978)

17. Chang, KC: Infinite Dimensional Morse Theory and Multiple Solution Problems. Birkhäuser, Boston (1993)

18. Rabinowitz, PH: Minimax Methods in Critical Point Theory with Applications to Differential Equations. CBMS, vol. 65 Am. Math. Soc, Providence (1986)

19. Li, SJ, Liu, JQ: Nontrivial critical point for asymptotically quadratic functions. J. Math. Anal. Appl. 165, 333-345 (1992)

10.1186/1687-1847-2013-309

Cite this article as: Zhang: Multiplicity results on discrete boundary value problems with double resonance via variational methods. Advances in Difference Equations 2013, 2013:309 